\title{
Regulation of plant antiviral defense genes via host RNA-silencing mechanisms
}

\author{
Paola Leonetti ${ }^{1} \mathbb{B}$, Johannes Stuttmann ${ }^{2}$ (]) and Vitantonio Pantaleo ${ }^{1,3^{*}}$ (])
}

\begin{abstract}
Background: Plants in nature or crops in the field interact with a multitude of beneficial or parasitic organisms, including bacteria, fungi and viruses. Viruses are highly specialized to infect a limited range of host plants, leading in extreme cases to the full invasion of the host and a diseased phenotype. Resistance to viruses can be mediated by various passive or active mechanisms, including the RNA-silencing machinery and the innate immune system.

Main text: RNA-silencing mechanisms may inhibit viral replication, while viral components can elicit the innate immune system. Viruses that successfully enter the plant cell can elicit pattern-triggered immunity (PTI), albeit by yet unknown mechanisms. As a counter defense, viruses suppress PTI. Furthermore, viral Avirulence proteins (Avr) may be detected by intracellular immune receptors (Resistance proteins) to elicit effector-triggered immunity (ETI). ETI often culminates in a localized programmed cell death reaction, the hypersensitive response (HR), and is accompanied by a potent systemic defense response. In a dichotomous view, RNA silencing and innate immunity are seen as two separate mechanisms of resistance. Here, we review the intricate connections and similarities between these two regulatory systems, which are collectively required to ensure plant fitness and resilience.

Conclusions: The detailed understanding of immune regulation at the transcriptional level provides novel opportunities for enhancing plant resistance to viruses by RNA-based technologies. However, extensive use of RNA technologies requires a thorough understanding of the molecular mechanisms of RNA gene regulation. We describe the main examples of host RNA-mediated regulation of virus resistance.
\end{abstract}

Keywords: Resistance, dsRNAs, VAMPs, PRRs, NLRs, Broad-spectrum resistance, RNA silencing

\section{Background}

Plants encounter numerous microorganisms and other higher living organisms throughout their life span. Some are beneficial, and even symbiotic. Others are harmful and can cause disease and death of the host if a prompt defense reaction or immunity is not triggered. The resilience of plants to environmental conditions and changes depends on their ability to promote beneficial interactions and activate defenses, when necessary, in a robust

\footnotetext{
*Correspondence: vitantonio.pantaleo@cnr.it

${ }^{1}$ Department of Biology, Agricultural and Food Sciences, Institute for Sustainable Plant Protection, Research Unit of Bari, CNR, 70126 Bari, Italy

Full list of author information is available at the end of the article
}

and energy-efficient manner. Based on this, the numerous mechanisms of molecular signal perception which are similar, yet distinct in downstream responses, during interactions with symbionts and pathogens, therefore require a coordinated machinery of control [1].

Viruses are potential plant pathogens and, therefore, will encounter plant's defense barriers at every step of their replication cycle; i.e. via (i) spread in the agro-ecosystems and transmission, (ii) plant cell infection, and (iii) systemic invasion. In regards to the host, the mechanisms against viral attacks can be summarized in: (i) nonhost resistance via physical barriers (e.g., waxy cuticles and/or thickened cell walls; preventing transmission by insect vectors), (ii) passive resistance in which the host blocks or lacks a component required by the virus to original author(s) and the source, provide a link to the Creative Commons licence, and indicate if changes were made. The images or other third party material in this article are included in the article's Creative Commons licence, unless indicated otherwise in a credit line to the material. If material is not included in the article's Creative Commons licence and your intended use is not permitted by statutory regulation or exceeds the permitted use, you will need to obtain permission directly from the copyright holder. To view a copy of this licence, visit http://creativecommons.org/licenses/by/4.0/. The Creative Commons Public Domain Dedication waiver (http://creativeco mmons.org/publicdomain/zero/1.0/) applies to the data made available in this article, unless otherwise stated in a credit line to the data. 
complete its life cycle, (iii) pattern-triggered immunity (PTI), (iv) effector-triggered immunity (ETI), and (v) the RNA-silencing system. Several RNA-based regulatory mechanisms of genes involved in resistance to microbes have been identified so far [2]. RNA-based mechanisms offer the advantage of being readily reversible in absence of pathogens. RNA-based regulation has emerged as a critical layer of control in plant immunity also in the case of virus infection. This review presents an overview of RNA-based regulatory mechanisms as the main actors of plant antiviral immune responses.

\section{PTI-based antiviral responses}

Canonical PTI is mediated by cell surface-localized pattern-recognition receptors (PRRs) that are either receptor-like kinases (RLKs) or receptor-like proteins. PRRs commonly recognize relatively conserved signature molecules characteristic for a whole class of organisms, referred to as microbe/pathogen-associated molecular patterns (MAMPs/PAMPs; reviewed in [3]). The best-characterized PAMP-PRR pair involves recognition of a 22-amino-acid epitope (flg22) derived from bacterial flagellin by the leucine-rich repeat (LRR) receptor kinase FLAGELLIN-SENSING 2 (FLS2). PAMP recognition triggers a cascade of reactions collectively forming the basal defense layer. PTI-induced reactions include $\mathrm{Ca}^{2+}$-influx, activation of mitogen-activated protein kinases, production of reactive oxygen species (ROS) and nitric oxide (NO), cell wall reinforcement, and salicylic acid (SA) synthesis and signalling $[4,5]$. Furthermore, PRRs may also detect host endogenous molecules released upon cell damage called damage-associated molecular patterns (DAMPs). During infection, many microbes translocate virulence factors (effectors) directly into the plant cell cytoplasm. One major function of effectors is the suppression of PTI responses, thus creating effector-triggered susceptibility [6]

Based on the firmly established definitions of microbial PAMPs and effectors [6,7] viruses are not generally considered as encoding PAMPs or effectors. Nonetheless, successful transmission and entry into a plant cell exposes a virus to the PTI defense layer, and recent reports suggest that classical plant PTI initiated by transmembrane PRRs limits virus infection. Pre-activation of PTI with non-viral PAMPs confers resistance to virus infection $[8,9]$, indicating that PTIinduced immune responses confer protection against viruses, and most viral genomes encode suppressors of PTI, similar to microbial effectors, i.e. the disease-specific protein of rice stripe virus (RSV) [10], cauliflower mosaic virus (CaMV) P6 [11] and plum pox virus capsid protein [12]. Upon PAMP perception, PTI signalling depends on an intricate network of co-receptors and receptor-like cytoplasmic kinases (reviewed, in [13]), such as the LRR-RLK BRASSINOSTEROID INSENSITIVE1 (BRI1)-ASSOCIATED KINASE 1 (BAK1/ SERK3) and related SOMATIC EMBRYOGENESIS RECEPTOR KINASES (SERKs). As exclusively intracellular pathogens, how viruses might be detected extracellularly by the surface-localized PRRs, remains an open question. However, it is conceivable that, similar to DAMPs, viral PAMPs (VAMPs) are exposed in the apoplast either actively or upon cellular damage. In line with such a scenario, the detection of protein and RNA components of turnip mosaic virus (TuMV) in the plant extracellular space during viral infection was reported [14]; this would partly solve the apparent contradiction and open novel avenues of investigations in plant virology.

Similarly, classical PRRs mediating resistance to viruses and corresponding VAMP-ligands remain to be identified. However, double-stranded (ds) RNAs, dsRNA-like molecules of viral origin (i.e. replication intermediates, highly structured single stranded RNA transcripts), the synthetic dsRNA analog polyinosinicpolycytidylic acid and bacterial RNA preparations can induce PTI responses in Arabidopsis [15, 16]. Intriguingly, dsRNA-induced PTI is independent of Dicer-like proteins (DCLs), but requires the LRR-RLK SERK1 [15], thus making it distinct from the well-characterized PTI induced by microbial elicitors and the RNA-silencing pathway. Furthermore, NSP-INTERACTING KINASE 1 (NIK1), which belongs to the same class of LRR-RLKs as BAK1, is strongly associated to resistance to begomoviruses, and is targeted by the viral nuclear shuttle protein during infection [17]. Constitutive activation of NIK1 leads to repression of genes coding for components of the translation machinery and global suppression of translation. Thus, although NIK1 is structurally related BAK1/SERK3, it induces an immune response distinct from those depending on this major PRR coreceptor to suppress viral replication.

Although dsRNA can thus apparently induce PTI, it also triggers the classical RNA-silencing pathway, which acts as the major virus resistance mechanism in plants. Comparing PTI and the RNA-silencing pathway, DCLs function in analogy to PRRs by binding dsRNA molecules, the VAMPs, in the cytoplasm (Fig. 1) [18]. Activation of DCL-dependent defense is associated with a massive production of endogenous viral-activated siRNAs (va-siRNAs) and a consequent widespread silencing of host genes [19, 20]. Accordingly, classical PTI and the RNA-silencing pathway can be considered to function in parallel for perception of VAMPs as a first layer of defense (Fig. 1). 


\begin{tabular}{|c|c|}
\hline $\begin{array}{c}\text { Sequence-specific } \\
\text { viral-siRNAs } \\
\text { guided } \\
\text { antiviral silencing }\end{array}$ & $\begin{array}{c}\text { Widespread suppression } \\
\text { of viral and } \\
\text { host genes expression }\end{array}$ \\
\hline
\end{tabular}<smiles>C1CC2CC1CC1CC1C2</smiles>

PTI PRRs

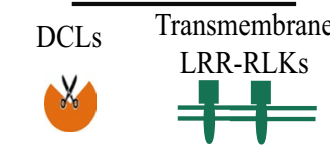

$\overbrace{\text { RNAi-based }}$

antiviral immunity

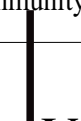

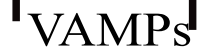

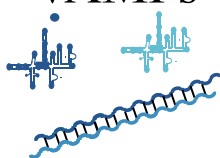

ETI

Local

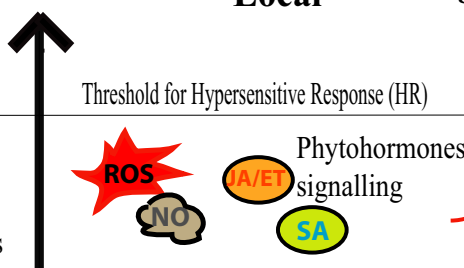

Virulence effectors
(i.e. VSR and other

antipathogen

responses

viral proteins )
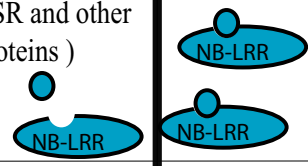

R-proteins

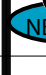

(1)

Threshold for Resistance

\section{$\mathrm{HIGH}$}

DEFENCE

\section{Systemic signals}

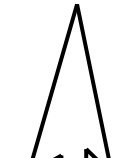

\section{I}

11

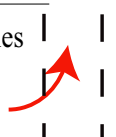

(1)

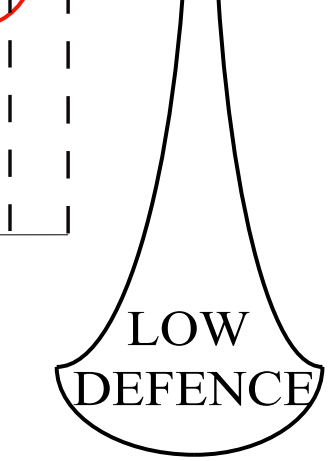

Fig. 1 "Zig-Zag" model by Jones and Dangl [6] adapted for representing plant defense responses to viruses. Virus-associated molecular patterns, VAMPs, typically double-stranded (ds)RNA of viral origin, can induce either RNAi-based antiviral or a canonical pathogen triggered immunity (PTI) upon recognition by Dicer-like proteins (DCLs) or transmembrane leucine rich pattern-recognition receptors (PRRs), respectively. The PTI response evolves into sequence-specific antiviral silencing and/or into wide-spread suppression of host gene expression. Viruses express virulence effectors (i.e., viral suppressors of RNA silencing, VSRs) that can suppress PTI and lower the level of resistance. In resistant plant accessions, virulence effectors or virus structural components are recognised by intracellular immune receptors, commonly of the NB-LRR type. Immune receptor activation triggers production of reactive oxygen species (ROS), NO, phytohormone signalling (Jasmonate, JA; Ethylene, ET; salicylic acids, SA) and the hypersensitive responses (HR). ETI further induces systemic acquired resistance (SAR)

\section{ETI-based antiviral responses}

In view of the above considerations, PTI was only recently included into virus-host interaction models, whereas ETI has long been recognized as an efficient plant defense mechanism against viruses. ETI is considered as a second layer of the plant innate immune system: effector proteins, delivered into host cells by adapted plant pathogens to suppress PTI, can become recognized by intracellular immune receptors in resistant host isolates. Effector recognition and immune receptor activation induce the rapid and efficient ETI response [6]. As an extreme output, ETI can culminate in the hypersensitive response, a programmed cell death reaction at infection sites thought to limit pathogen spread. Most intracellular immune receptors belong to the nucleotide binding/leucine-rich repeat (NLR) class of proteins reviewed in e.g. [21, 22]. Although the term effectors is not classically used in plant virus interactions, also viruses encode virulence factors or structural components, which can become recognized in an accession- and isolate-specific manner by the ETI system (reviewed in [23, 24]) (Fig. 1).

Over the past decade, several $R$ genes that mediate dominant resistance against viruses have been identified (reviewed in [25]). Viral components recognized by $\mathrm{R}$ proteins are diverse, and include coat protein, movement protein, helicase or others. Plant NLRs are divided in two major groups based on their $\mathrm{N}$-terminal coiled coil $(\mathrm{CC})$ or Toll-like interleukin 1 receptor (TIR) domains, and are referred to as CNLs and TNLs, respectively [26]. Both these major classes of NLRs can function in resistance to viruses. Recent insights suggest that CNLs can induce ETI directly, by formation of a resistosome complex upon activation, which inserts into membranes to function as a $\mathrm{Ca}^{2+}$-permeable ion 
channel [27, 28]. By contrast, TNLs assemble into holoenzymes with NADase activity upon activation $[29,30]$. Downstream of NADase activity, TNL immunity depends on ENHANCED DISEASE SUSCEPTIBILITY1 (EDS1) complexes and helper NLRs termed RNLs [31-34]. RNLs incorporate a subtype of the $\mathrm{CC}$ domain, the $\mathrm{CC}_{\mathrm{R}}$ or $\mathrm{CC}_{\mathrm{HeLo}}$ domain, with similarity to that of the non-LRR protein RESISTANCE TO POWDERY MILDEW $8[26,35]$. While the $C_{R}$-type helper NLRs are relatively conserved, TNLs and CNLs belong to the most variable and most rapidly evolving gene families in plants [36-38]. ETI is induced only upon presence of an immune receptor in the host (encoded by the $R$ gene) and the corresponding, recognized component in the pathogen (encoded by the Avr gene). Thus, ETI-mediated virus resistance generally is dominant and monogenic. Intracellular immune receptors can either recognize non-self as direct binders, or modified self as guardian NLRs $[39,40]$. In the case of resistance to viruses, the details of pathogen recognition have been analysed in only few cases, as e.g. the recognition of the tobacco mosaic virus p50 helicase domain by the tobacco $\mathrm{N}$ receptor [41]. ETI against plant viruses often results in the hypersensitive response and extreme resistance, and is accompanied by phytohormone release [42]. By subsequent long-distance signalling, ETI therefore not only comprises the local response at the infection site, but also a systemic response conferring resistance to subsequent infections, known as systemic acquired resistance (SAR). SAR is conserved across different plant families and is non-specific. Therefore, it is effective against different pathogens (viruses, fungi, and bacteria) and may confer protection to subsequent infections.

\section{Main text}

In this section we describe the main examples of host RNA-mediated regulation of virus resistance factors playing a role in PTI and ETI.

\section{RNA-based regulation of dicers: binding of VAMPs in analogy to PRRs}

MicroRNAs (miRNAs) associate with Argonaute (AGO) proteins to direct widespread post-transcriptional gene repression. Arabidopsis miR393 was the first miRNA implicated in bacterial PTI [43]. The miR393 gene is transcriptionally activated by flg22. High miR393 levels repress accumulation of the transcripts of the auxin receptor TRANSPORT INHIBITOR RESPONSE 1 (TIR1) and related AUXIN-SIGNALING F-BOX (AFB) proteins. This leads to reduced ubiquitination-mediated turnover of the auxin/indole-3-acetic acid (Aux/IAA) co-repressors, and thus suppression of auxin-responsive gene expression and PTI defenses. Indeed, bacterial growth was reduced in miR393 overexpressing Arabidopsis lines [43].

Similarly, TIR1-mediated Aux/IAA turnover and auxin signalling were found to be significantly affected in rice during infection by rice black-streaked dwarf virus (RBSDV) [44]. However, in contrast to flg22 recognition, miR393 is not upregulated in RBSDV-infected rice. This suggests that repression of auxin signalling by RBSDV may occur by a distinct mechanism not involving PRRPAMP recognition. In the case of rice dwarf virus (an RBSDV relative), auxin signalling seems to be altered by the capsid protein $\mathrm{P} 2$ via a protein-protein interaction with at least one AUX/IAA, rather than by RNA-based mechanisms [45]. Accordingly, antiviral PRRs sensu stricto remain to be identified (although NIK1 may represent a valid candidate in this respect), and PRR involvement in antiviral PTI has been suggested only by indirect evidences.

However, given that RNAi-mediated antiviral immunity acts as the major virus resistance mechanism in plants and exhibits PTI features [11], the main role of PRRs is played by plant DCLs (Figs. 1 and 2). Plant DCLs have specialized functions in producing short RNAs of 21- to 24-nucleotides (nt), including miRNAs and small-interfering (si)RNAs of endogenous or viral origin (vsiRNAs) [46]. DCL1-derived miRNAs modulate the expression of antiviral DCLs upon perception of viral infections (Fig. 2). As a consequence, antiviral DCLderived vsiRNAs program antiviral effectors and confer antiviral immunity. In Arabidopsis, antiviral DCLs, i.e. DCL4 and DCL2, act redundantly in antiviral immunity: DCL4 and DCL2 are both sufficient for blocking the virus from spreading in plant tissues. However, viral suppressors of RNA-silencing (VSRs) reveal non-redundant functions and sub-specialization among DCL2/4 by specific inhibition of DCL4 activity [47, 48]. In vivo data revealed that miRNA sequestration by VSRs is most relevant at the early stage of an infection prior to the emergence of virus-induced symptoms or spread [49]. In the case of p19-deficient cymbidium ringspot virus, miR162 downregulates the expression of DCL1, which keeps the miRNA expression levels low and the DCL4 levels high. Conversely, early in infection, p19 binds miR403 and the expression of the antiviral AGO2 is increased, whereas the low affinity of p19 for AGO1-regulating miR168 ensures homeostasis of AGO1 [49] (Fig. 2).

The basis for the DCL1-dependent negative regulation of DCL4 levels remains to be established. However, a novel regulatory mechanism of the antiviral DCL2 was recently revealed (Fig. 3a). DCL2 plays a key role in producing 22-nt sRNAs from endogenous mRNAs, viral RNAs and from transgenes when other DCLs, especially 


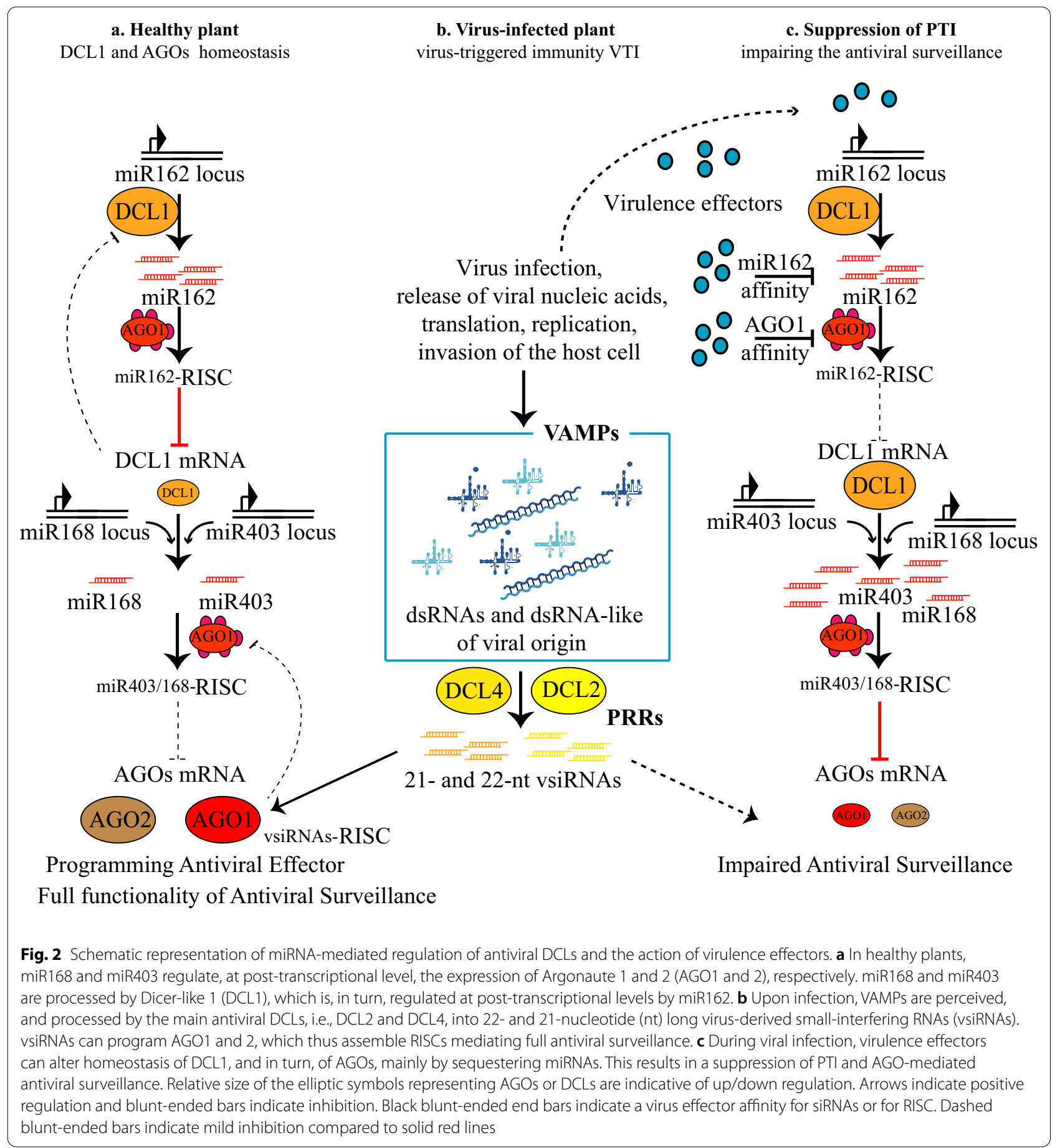

DCL4, are absent [46, 50-52]. DCL2 has other roles in the systemic spread of transitive silencing between cells and the vascular system [53, 54]. In tomato, DCL2 is the major dicer of defense against tobacco mosaic virus (TMV) and potato virus $\mathrm{X}$, which is mediated by an unusual miRNA-dependent mechanism [55]. It is widely accepted that DCL1 typically produces 21-nt miRNAs, but generates 22-nt products if the precursor RNA has an asymmetric bulge in the base-paired region [56]. However, an alternative mechanism for production of 22-nt sRNA production involves DCL2, and is independent of bulges in the precursor RNA. E.g., in tomato, the biogenesis of non-canonical 22-nt miR6026 is DCL2dependent [55]. Indeed, the miR6026 level is lower than 


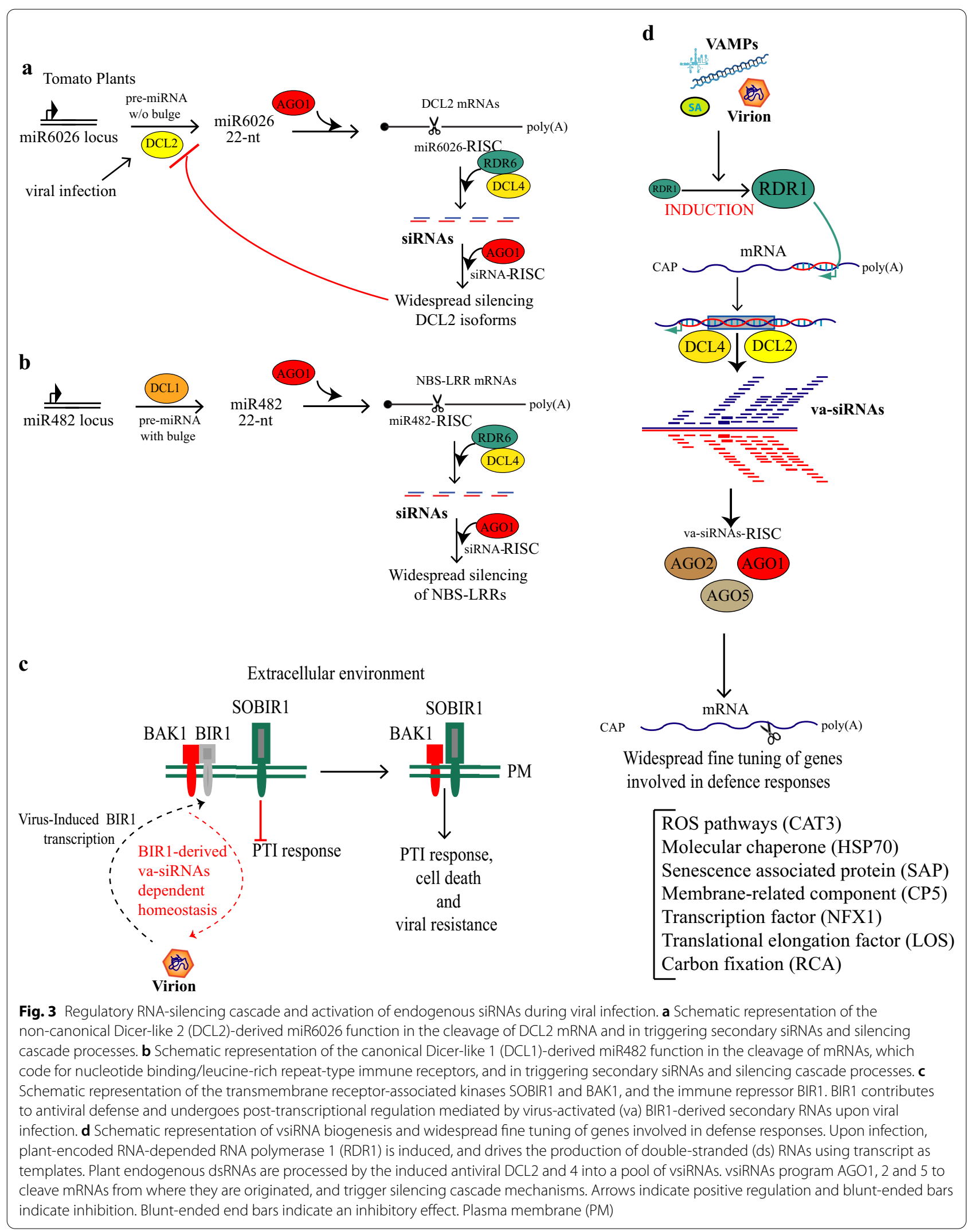


for wild-type in $d c l 2$-deficient plants, whereas the 22-nt miR482 level is unaffected except in $d c l 1$-deficient lines [55] (Fig. 3). The same authors also showed that the $D C L 2$ transcript isoforms are among the miR6026 target mRNAs. In Arabidopsis, the 22-nt miRNAs trigger secondary sRNA production using their mRNA targets as template [57]. Similarly, in tomato, sRNAs corresponded to the miR6026 targets, were partly DCL4 and RDR6dependent [55]. The antiviral activity of DCL2 was confirmed by the sRNA profiles of TMV-infected tomato: in $d c l 2$-deficient plants, the 22-nt viral sRNAs were less abundant than in wild-type.

\section{RNA-based regulation of NLR genes}

The RNA-silencing pathway further controls plant immune capacities via the ETI system. E.g., in tomato, levels of the NLR-type immune receptor slTM2, involved in antiviral ETI, are controlled by the 22-nt miR6026. Conversely to DCL2 mRNAs, slTM2 mRNA regulation is apparently not dependent on miR6026 targeting [55]. The authors attributed this to the fact that other miRNAs target the slTM2 mRNA and could trigger secondary siRNA in the absence of miR6026.

Expression of $N L R$ genes needs to be tightly controlled, as NLRs can also trigger autoimmunity in the absence of pathogen infection and inhibit plant growth; referred to as the "growth-defense trade-off" [58-60]. Therefore, plants have evolved sophisticated RNA-silencing-based cascade mechanisms to downregulate entire families of $N L R$ genes, thus preventing autoimmunity, and opening the "gates" to establish symbiosis [61]. miRNAs are widely accepted as master regulators of mRNAs of the NLR immune receptor gene family via the production of phased secondary siRNAs [62-66]. In tomato, the initiators of the cascade are 22-nt-long miRNAs of the miR482 superfamily [67] (Fig. 3b). Members of this superfamily vary in sequence and abundance in different plant species, but generally target the coding sequence of the P-loop motif within the mRNA sequences of NLR immune receptors [67]. Oftentimes, due to natural variation within NLRs, miRNA-mediated cleavage is affected by indels in NLR alleles [65]. The targeting of NLR transcripts causes mRNA decay and RDR6-dependent production of phased secondary siRNAs in register with the cleavage site, which can regulate further transcripts upon incorporation into the RNA-induced silencing complex (RISC) [68]. At least one NLR-derived secondary siRNA was shown to act in trans and target mRNAs of additional defense-related proteins. Further, secondary siRNAs acting in cis fuel the cascade mechanism and boost the down-regulation of $N L R$ genes. Viruses or bacteria can suppress sRNA-mediated NLR regulation by preventing miRNA incorporation into RISCs. As a result, a pathogen-induced expression of NLRs occurs and broadspectrum defense mechanisms are activated $[67,69]$.

The requirement for dynamic regulation of $N L R$ gene expression is further emphasized by characterization of miR1885 in Brassica rapa [70]. Here, miR1885 is not conserved, but appears to have recently evolved from an inverted duplication of an NLR gene. miR1885 expression is specifically induced upon TuMV infection, and regulates expression of highly sequence-related $T N L$ genes [70]. However, miR1885 also targets the trans-acting silencing (TAS) gene BraTIR1 [71]. In the absence of TuMV infection, miR1885 levels remain low to maintain normal development and basal immunity. Upon TuMV infection, repression of BraTIR1 is entailed by repression of the photosynthesis-related gene $\mathrm{BraCP} 24$, which subsequently accelerates floral transition [71]. This sophisticated mechanism illustrates how plants, besides actively mounting defenses via the PTI, ETI and RNA-silencing pathways, invented alternative solutions to rapidly escape from virus infection by transitioning to a safe and healthy next generation.

\section{Widespread silencing of host genes and broad spectrum immunity}

BAK1-INTERACTING RECEPTOR-LIKE KINASE 1 (BIR1) was identified as a negative regulator of plant immunity and cell death in a systematic reverse genetic screen, and shown to interact with the PRR co-receptor BAK1 [72]. However, BIR1 overexpression also leads to severe developmental defects and triggers the activation of plant defenses [73]. During tobacco rattle virus (TRV) infection in Arabidopsis, BIR1 gene expression is activated in a partly salicylic acid-dependent manner, and is an important regulator of antiviral defenses [74]. Interestingly, siRNAs originating from BIR1 mRNA were found to be produced during TRV infection and are involved in BIR1 homeostasis [74] (Fig. 3c). Similar regulatory mechanisms may apply for additional plant immune components. Indeed, viral infections are accompanied by a massive production of siRNAs of plant origin, such as va-siRNAs, which drive the widespread silencing of host-gene expression [19, 20]. Data on BIR1 regulation in the TRV-Arabidopsis system extend observations from cucumber mosaic virus (CMV) and TuMV-infected Arabidopsis and CaMV-infected Brassicaeae.

An important finding derived from the discovery of va-siRNAs is that the investigated Brassicaceae species display a highly conserved va-siRNA induction response during infection with taxonomically unrelated viruses $[19,20]$. Moreover, the two antiviral dicers, DCL2 and DCL4, are mainly involved in the generation of vasiRNAs. va-siRNAs commonly target genes involved in biotic and abiotic stress response (Fig. 3d). Taken 
together, these observations support the notion that induction of antiviral silencing confers broad-spectrum antiviral activity as a result of widespread silencing of host genes (in particular negative regulators of defence responses), mediated by va-siRNAs in addition to specific antiviral defenses by vsiRNAs.

In the case of canonical PTI and PAMP perception, PRRs initiate signalling pathways leading to ROS production. Recent investigations (highlighted in [75]) have revealed that RNA-based mechanisms tune ROS pathways during plant virus infections. Interestingly, among the genes targeted by va-siRNAs and significantly downregulated in CaMV-infected Brassicaceae and, similarly, in $A$. thaliana infected by CMV- $\Delta 2 \mathrm{~b}$ (a CMV which lacks the $2 \mathrm{~b}$ VSR), there were reactive oxygen species (ROS) scavenging factors such as Catalase 3 (Cat3), which could represent an element of parallelism with canonical PTI [20]. ROS pathways can also be regulated by viral proteins (i.e. VSRs), which then mask RNA-based regulatory mechanisms. The case of CAT3 is emblematic: the interaction between CMV 2b and CAT3 seemed to be important to induce necrosis, as a consequence of CAT3 degradation via the proteasome pathway. Furthermore, CMV accumulates more abundantly in plants that do not express CAT3 [76]. It therefore appears that viruses hijack the host's ROS generation mechanism during infection to promote viral replication by va-siRNAsmediated gene regulation or viral protein-mediated CAT3 degradation. Another case worthy of note is that of a red clover necrotic mosaic virus protein that associates with positive regulators of the ROS production machinery. As a result ROS are induced by the plant and viral replication is more abundant [77]. In the same study, the authors have found a similar evidence for brome mosaic virus replication.

In rice infected with RSV, miR528 is suppressed at both the transcriptional and post-transcriptional levels. Furthermore, RSV induces the accumulation of the non-catalytic AGO18. AGO18 sequesters miR528 away from AGO1 to block the formation of an effective RISC. These events induce the accumulation of the miR528 target, L-ascorbate oxidase, thereby regulating cellular redox status and priming ROS-mediated resistance against RSV infection [78]. Based on the notion that ROS could limit virus invasion, the overexpression of negative regulators of ROS production could result in enhanced viral replication. Triticum aestivum thioredoxin-like gene (TaAAED1) encodes a negative regulator of ROS production in the chloroplast. Wheat yellow mosaic virus-derived dsRNAs are perceived and processed by DCL4 to produce 21-nt vsiRNAs. Upon incorporation into the RISC, the vsiRNAs suppress the expression of TaAAED1 in a dose-dependent (more viral RNA, more downregulation) and sequencespecific manner. Therefore, chloroplast-generated ROS are known to induce retrograde signalling (from the chloroplast to the nucleus), leading to the modulation of the expression of pathogenesis-related genes that are involved in the defense response to viruses [79].

All together, these studies demonstrate that plant viruses are causal agents of ROS induction in infected plants; however, the function of ROS in plant-virus interactions remains unclear, because despite the intuitive idea that ROS are plant defence tools, in many cases intracellular bursts of ROS have been associated with increased viral fitness.

\section{Conclusions}

Recent discoveries emphasize consistent parallels and connections between canonical plant immunity mechanisms (PTI, ETI) and the RNA-silencing pathway in orchestrating resistance to viruses and microbes. Especially the PTI system has been previously well-studied for its role and molecular functions in plant-microbe interactions, which may help to decipher its role in virus resistance in future. A most relevant finding is that DCLs may be the main actors, able to perceive viral PAMPs (VAMPs), in analogy to PRRs, and trigger resistance. These findings are extendable to mammalian cells where RNA detection mechanisms are well-established [80]. Recent insights offer important perspectives for bolstering plant defense against bacterial and fungal pathogens via RNAi approaches or applications. Studies of virushost interactions show how RNA-mediated gene regulation can confer broad-spectrum resistance efficiently even towards pathogens that are routinely controlled by pesticides.

\footnotetext{
Abbreviations

PTI: Pattern-triggered immunity; Avr: Avirulence proteins; ETI: Effectortriggered immunity; HR: Hypersensitive response; PRR: Pattern-recognition receptor; RLK: Receptor-like kinases; MAMPs/PAMPs: Microbe/pathogen-associated molecular patterns; LRR: Leucine-rich repeat; FLS2: FLAGELLIN-SENSING 2 (FLS2); ROS: Reactive oxygen species; NO: Nitric oxide; SA: Salicylic acid; DAMPs: Damage-associated molecular patterns (DAMPs); BAK1/SERK3: BRASSINOSTEROID INSENSITIVE1 (BRI1)-ASSOCIATED KINASE 1; SERKs: SOMATIC EMBRYOGENESIS RECEPTOR KINASES; VAMPs: Viral PAMPs; TUMV: Turnip mosaic virus; dsRNAs: Double-stranded RNAs; DCLs: Dicer-like proteins; NIK1: NSPINTERACTING KINASE 1; va-siRNAs: Viral-activated siRNAs; R-proteins: Resistance proteins; NLR: Nucleotide binding/leucine-rich repeat; CC: Coiled coil; TIR: Toll-interleukin 1 receptor; CNLs: CC-NLRs; TNLs: TIR-NLRs; NADase: Nicotinamide adenine dinucleotide glycohydrolase; SAR: Systemic acquired resistance; miRNA: MicroRNA; AGO: Argonaute; TIR1: TRANSPORT INHIBITOR RESPONSE 1; AFB: AUXIN-SIGNALING F-BOX; Aux/IAA: Auxin/indole-3-acetic acid; RBSDV: Rice black-streaked dwarf virus; nt: Nucleotides; siRNAs: Small-interfering RNA; vsiRNAs: Viral-derived siRNA; VSR: Viral suppressor of RNA-silencing; TMV: Tobacco mosaic virus; RISC: RNA-induced silencing complex; TUMV: Turnip mosaic virus; TAS: Trans-acting silencing; BIR1: BAK1-INTERACTING RECEPTORLIKE KINASE 1;TRV: Tobacco rattle virus; CaMV: Cauliflower mosaic virus; TaAAED1: Triticum aestivum thioredoxin-like Gene.
} 


\section{Acknowledgements}

We thank Prof. Padubidri V Shivaprasad for critical reading of the present work. Thanks to CNR-STM 2020 (VP) and to Martin Luther University for hosting VP as guest professor and allowing extended discussions.

\section{Authors' contributions}

VP organized the content; VP and PL wrote the first draft of the manuscript, VP. $P L$, IS edited the manuscript. VP and PL generated the figures. All authors read and approved the final manuscript.

\section{Funding}

Not applicable.

\section{Availability of data and materials}

Not available.

\section{Declarations}

Ethics approval and consent to participate

Not applicable.

\section{Consent for publication}

Not applicable.

\section{Competing interests}

The authors declare that they have no competing interests.

\section{Author details}

'Department of Biology, Agricultural and Food Sciences, Institute for Sustainable Plant Protection, Research Unit of Bari, CNR, 70126 Bari, Italy. ${ }^{2}$ Institute of Biology, Department of Plant Genetics, Martin Luther University, Halle-Wittenberg, 06120 Halle (Saale), Germany. ${ }^{3}$ Institute of Biochemistry and Biotechnology, Martin Luther University, Halle-Wittenberg, 06120 Halle (Saale), Germany.

\section{Received: 10 August 2021 Accepted: 14 September 2021}

Published online: 26 September 2021

\section{References}

1. Zipfel C, Oldroyd GE. Plant signalling in symbiosis and immunity. Nature. 2017;543:328-36.

2. Staiger D, Korneli C, Lummer M, Navarro L. Emerging role for RNA-based regulation in plant immunity. New Phytol. 2013;197:394-404.

3. Boutrot F, Zipfel C. Function, discovery, and exploitation of plant pattern recognition receptors for broad-spectrum disease resistance. Annu Rev Phytopathol. 2017;55:257-86.

4. Zipfel C, Robatzek S, Navarro L, Oakeley EJ, Jones JDG, Felix G, et al. Bacterial disease resistance in Arabidopsis through flagellin perception. Nature. 2004;428:764-7.

5. Navarro L, Zipfel C, Rowland O, Keller I, Robatzek S, Boller T, et al. The transcriptional innate immune response to flg22. Interplay and overlap with Avr gene-dependent defense responses and bacterial pathogenesis. Plant Physiol. 2004:135:1113-28.

6. Jones JD, Dangl JL. The plant immune system. Nature. 2006;444:323-9.

7. Spoel SH, Dong X. How do plants achieve immunity? Defence without specialized immune cells. Nat Rev Immunol. 2012;12:89-100.

8. Li B, Ferreira MA, Huang M, Camargos LF, Yu X, Teixeira RM, et al. The receptor-like kinase NIK1 targets FLS2/BAK1 immune complex and inversely modulates antiviral and antibacterial immunity. Nat Commun. 2019;10:4996.

9. Kørner CJ, Klauser D, Niehl A, Domínguez-Ferreras A, Chinchilla D, Boller $T$, et al. The immunity regulator BAK1 contributes to resistance against diverse RNA viruses. Mol Plant-Microbe Interactions. 2013;26:1271-80.

10. Kong L, Wu J, Lu L, Xu Y, Zhou X. Interaction between Rice stripe virus disease-specific protein and host PsbP enhances virus symptoms. Mol Plant. 2014;7:691-708

11. Zvereva AS, Golyaev V, Turco S, Gubaeva EG, Rajeswaran R, Schepetilnikov $M V$, et al. Viral protein suppresses oxidative burst and salicylic acid-dependent autophagy and facilitates bacterial growth on virusinfected plants. New Phytol. 2016;211:1020-34.

12. Nicaise V, Candresse T. Plum pox virus capsid protein suppresses plant pathogen-associated molecular pattern (PAMP)-triggered immunity: a plant virus effector impairs PTI early responses. Mol Plant Pathol. 2017;18:878-86.

13. Saijo Y, Loo EP, Yasuda S. Pattern recognition receptors and signaling in plant-microbe interactions. Plant J. 2018;93:592-613.

14. Movahed N, Cabanillas DG, Wan J, Vali H, Laliberté J-F, Zheng H. Turnip mosaic virus components are released into the extracellular space by vesicles in infected leaves. Plant Physiol. 2019;180:1375-88.

15. Niehl A, Wyrsch I, Boller T, Heinlein M. Double-stranded RNAs induce a pattern-triggered immune signaling pathway in plants. New Phytol. 2016:211:1008-19.

16. Lee B, Park Y, Lee S, Song GC, Ryu C. Bacterial RNAs activate innate immunity in Arabidopsis. New Phytol. 2016;209:785-97.

17. Zorzatto C, Machado JPB, Lopes KVG, Nascimento KJT, Pereira WA, Brustolini OJB, et al. NIK1-mediated translation suppression functions as a plant antiviral immunity mechanism. Nature. 2015;520:679-82.

18. Amari $\mathrm{K}$, Niehl A. Nucleic acid-mediated PAMP-triggered immunity in plants. Curr Opin Virol. 2020;42:32-9.

19. Cao M, Du P, Wang X, Yu YQ, Qiu YH, Li W, et al. Virus infection triggers widespread silencing of host genes by a distinct class of endogenous siRNAs in Arabidopsis. Proc Natl Acad Sci USA. 2014;111:14613-8.

20. Leonetti P, Ghasemzadeh A, Consiglio A, Gursinsky T, Behrens S, Pantaleo $\checkmark$. Endogenous activated small interfering RNAs in virus-infected Brassicaceae crops show a common host gene-silencing pattern affecting photosynthesis and stress response. New Phytol. 2021;229:1650-64.

21. Cui H, Tsuda K, Parker JE. Effector-triggered immunity: from pathogen perception to robust defense. Annu Rev Plant Biol. 2015;66:487-511.

22. Bi G, Zhou J-M. Regulation of cell death and signaling by pore-forming resistosomes. Annu Rev Phytopathol. 2021;59:239-63.

23. Nakahara KS, Masuta C. Interaction between viral RNA silencing suppressors and host factors in plant immunity. Curr Opin Plant Biol. 2014:20:88-95.

24. Mandadi KK, Scholthof KB. Plant immune responses against viruses: how does a virus cause disease? Plant Cell. 2013;25:1489-505.

25. de Ronde D, Butterbach P, Kormelink R. Dominant resistance against plant viruses. Front Plant Sci. 2014;5:307.

26. Lapin D, Bhandari DD, Parker JE. Origins and immunity networking functions of EDS1 family proteins. Annu Rev Phytopathol. 2020;58:253-76.

27. Wang J, Hu M, Wang J, Oi J, Han Z, Wang G, et al. Reconstitution and structure of a plant NLR resistosome conferring immunity. Science. 2019;364:eaav5870.

28. Bi G, Su M, Li N, Liang Y, Dang S, Xu J, et al. The ZAR1 resistosome is a calcium-permeable channel triggering plant immune signaling. Cell. 2021;184:3528-41.

29. Martin R, Qi T, Zhang H, Liu F, King M, Toth C, et al. Structure of the activated ROQ1 resistosome directly recognizing the pathogen effector XopQ. Science. 2020;370:eabd9993.

30. Ma S, Lapin D, Liu L, Sun Y, Song W, Zhang X, et al. Direct pathogeninduced assembly of an NLR immune receptor complex to form a holoenzyme. Science. 2020;370:eabe3069.

31. Jubic LM, Saile S, Furzer OJ, El Kasmi F, Dangl JL. Help wanted: helper NLRs and plant immune responses. Curr Opin Plant Biol. 2019;50:82-94.

32. Lapin D, Kovacova V, Sun X, Dongus JA, Bhandari D, von Born P, et al. A coevolved EDS1-SAG101-NRG1 module mediates cell death signaling by TIR-domain immune receptors. Plant Cell. 2019;31:2430-55.

33. Wagner S, Stuttmann J, Rietz S, Guerois R, Brunstein E, Bautor J, et al. Structural basis for signaling by exclusive EDS1 heteromeric complexes with SAG101 or PAD4 in plant innate immunity. Cell Host Microbe. 2013;14:619-30.

34. Gantner J, Ordon J, Kretschmer C, Guerois R, Stuttmann J. An EDS1SAG101 complex is essential for TNL-mediated immunity in nicotiana benthamiana. Plant Cell. 2019;31:2456-74.

35. Xiao S, Ellwood S, Calis O, Patrick E, Li T, Coleman M, et al. Broad-spectrum mildew resistance in Arabidopsis thaliana mediated by RPW8. Science. 2001;291:118-20.

36. Collier SM, Hamel L-P, Moffett P. Cell death mediated by the N-terminal domains of a unique and highly conserved class of NB-LRR protein. Mol Plant-Microbe Interactions. 2011:24:918-31. 
37. Jacob F, Vernaldi S, Maekawa T. Evolution and conservation of plant NLR functions. Front Immunol. 2013;4:297.

38. Prigozhin DM, Krasileva KV. Analysis of intraspecies diversity reveals a subset of highly variable plant immune receptors and predicts their binding sites. Plant Cell. 2021;33:998-1015.

39. Kourelis J, van der Hoorn RAL. Defended to the nines: 25 years of resistance gene cloning identifies nine mechanisms for $\mathrm{R}$ protein function. Plant Cell. 2018;30:285-99.

40. Cesari S. Multiple strategies for pathogen perception by plant immune receptors. New Phytol. 2018;219:17-24.

41. Burch-Smith TM, Schiff M, Caplan JL, Tsao J, Czymmek K, Dinesh-Kumar SP. A novel role for the TIR domain in association with pathogen-derived elicitors. PLoS Biol. 2007;5:e68.

42. Carr JP, Lewsey MG, Palukaitis P. Signaling in induced resistance. Adv Virus Res. 2010;76:57-121.

43. Navarro L, Dunoyer P, Jay F, Arnold B, Dharmasiri N, Estelle M, et al. A plant miRNA contributes to antibacterial resistance by repressing auxin signaling. Science. 2006:312:436-9.

44. Sun Z, He Y, Li J, Wang X, Chen J. Genome-wide characterization of rice black streaked dwarf virus-responsive MicroRNAs in rice leaves and roots by small RNA and degradome sequencing. Plant Cell Physiol. 2015;56:688-99.

45. Jin L, Qin Q, Wang Y, Pu Y, Liu L, Wen X, et al. Rice dwarf virus P2 protein hijacks Auxin signaling by directly targeting the rice OsIAA10 protein, enhancing viral infection and disease development. PLoS Pathog. 2016;12:e1005847.

46. Xie Z, Johansen LK, Gustafson AM, Kasschau KD, Lellis AD, Zilberman D, et al. Genetic and functional diversification of small RNA pathways in plants. PLoS Biol. 2004;2:E104.

47. Deleris A, Gallego-Bartolome J, Bao J, Kasschau KD, Carrington JC, Voinnet O. Hierarchical action and inhibition of plant Dicer-like proteins in antiviral defense. Science. 2006;313:68-71.

48. Azevedo J, Garcia D, Pontier D, Ohnesorge S, Yu A, Garcia S, et al. Argonaute quenching and global changes in Dicer homeostasis caused by a pathogen-encoded GW repeat protein. Genes Dev. 2010;24:904-15.

49. Pertermann R, Tamilarasan S, Gursinsky T, Gambino G, Schuck J, Weinholdt $C$, et al. A viral suppressor modulates the plant immune response early in infection by regulating MicroRNA activity. mBio. 2018;9:e00419-18.

50. Gasciolli V, Mallory AC, Bartel DP, Vaucheret H. Partially redundant functions of Arabidopsis DICER-like enzymes and a role for DCL4 in producing trans-acting siRNAs. Curr Biol. 2005;15:1494-500.

51. Bouche N, Lauressergues D, Gasciolli V, Vaucheret H. An antagonistic function for Arabidopsis DCL2 in development and a new function for DCL4 in generating viral siRNAs. EMBO J. 2006;25:3347-56.

52. Mlotshwa S, Pruss GJ, Peragine A, Endres MW, Li J, Chen X, et al. DICERLIKE2 plays a primary role in transitive silencing of transgenes in Arabidopsis. PLoS One. 2008;3:e1755.

53. Taochy C, Gursanscky NR, Cao J, Fletcher SJ, Dressel U, Mitter N, et al. A genetic screen for impaired systemic RNAi highlights the crucial role of DICER-LIKE 2. Plant Physiol. 2017;175:1424-37.

54. Wu Y-Y, Hou B-H, Lee W-C, Lu S-H, Yang C-J, Vaucheret H, et al. DCL2- and RDR6-dependent transitive silencing of SMXL4 and SMXL5 in Arabidopsis dcl4 mutants causes defective phloem transport and carbohydrate overaccumulation. Plant J. 2017;90:1064-78.

55. Wang Z, Hardcastle TJ, Canto Pastor A, Yip WH, Tang S, Baulcombe DC. A novel DCL2-dependent miRNA pathway in tomato affects susceptibility to RNA viruses. Genes Dev. 2018;32:1155-60.

56. Manavella PA, Koenig D, Weigel D. Plant secondary siRNA production determined by microRNA-duplex structure. Proc Natl Acad Sci USA. 2012:109:2461-6.

57. Cuperus JT, Carbonell A, Fahlgren N, Garcia-Ruiz H, Burke RT, Takeda A, et al. Unique functionality of 22-nt miRNAs in triggering RDR6-dependent siRNA biogenesis from target transcripts in Arabidopsis. Nat Struct Mol Biol. 2010:17:997-1003.

58. Ordon J, Martin P, Erickson JL, Ferik F, Balcke G, Bonas U, et al. Disentangling cause and consequence: genetic dissection of the DANGEROUS MIX2 risk locus, and activation of the DM2h NLR in autoimmunity. Plant J. 2021;106:1008-23.

59. Chae E, Bomblies K, Kim ST, Karelina D, Zaidem M, Ossowski S, et al. Species-wide genetic incompatibility analysis identifies immune genes as hot spots of deleterious epistasis. Cell. 2014;159:1341-51.
60. Karasov TL, Chae E, Herman JJ, Bergelson J. Mechanisms to mitigate the trade-off between growth and defense. Plant Cell. 2017;29:666-80.

61. Delaux P-M, Schornack S. Plant evolution driven by interactions with symbiotic and pathogenic microbes. Science. 2021;371:eaba6605.

62. Li F, Pignatta D, Bendix C, Brunkard JO, Cohn MM, Tung J, et al. MicroRNA regulation of plant innate immune receptors. Proc Natl Acad Sci. 2012:109:1790-5.

63. Zhai J, Jeong DH, De Paoli E, Park S, Rosen BD, Li Y, et al. MicroRNAs as master regulators of the plant NB-LRR defense gene family via the production of phased, trans-acting siRNAs. Genes Dev. 2011;25:2540-53.

64. Fei Q, Li P, Teng C, Meyers BC. Secondary siRNAs from Medicago NB-LRRs modulated via miRNA-target interactions and their abundances. Plant J. 2015;83:451-65.

65. Chiumenti M, Catacchio CR, Miozzi L, Pirovano W, Ventura M, Pantaleo V. A short indel-lacking-resistance gene triggers silencing of the photosynthetic machinery components through TYLCSV-associated endogenous siRNAs in tomato. Front Plant Sci. 2018;9:1470.

66. Sós-Hegedűs A, Domonkos Á, Tóth T, Gyula P, Kaló P, Szittya G. Suppression of NB-LRR genes by miRNAs promotes nitrogen-fixing nodule development in Medicago truncatula. Plant Cell Environ. 2020;43:1117-29.

67. Shivaprasad PV, Chen HM, Patel K, Bond DM, Santos BA, Baulcombe DC. A microRNA superfamily regulates nucleotide binding site-leucine-rich repeats and other mRNAs. Plant Cell. 2012;24:859-74.

68. Vazquez F, Hohn T. Biogenesis and biological activity of secondary siRNAs in plants. Scientif. (Cairo). 2013;2013:783253.

69. Canto-Pastor A, Santos BAMC, Valli AA, Summers W, Schornack S, Baulcombe DC. Enhanced resistance to bacterial and oomycete pathogens by short tandem target mimic RNAs in tomato. Proc Natl Acad Sci. 2019;116:2755-60.

70. He XF, Fang YY, Feng L, Guo HS. Characterization of conserved and novel microRNAs and their targets, including a TuMV-induced TIR-NBS-LRR class R gene-derived novel miRNA in Brassica. FEBS Lett. 2008;582:2445-52.

71. Cui C, Wang JJ, Zhao JH, Fang YY, He XF, Guo HS, et al. A Brassica miRNA regulates plant growth and immunity through distinct modes of action. Mol Plant. 2020;13:231-45.

72. Gao M, Wang X, Wang D, Xu F, Ding X, Zhang Z, et al. Regulation of cell death and innate immunity by two receptor-like kinases in Arabidopsis. Cell Host Microbe. 2009:6:34-44.

73. Liebrand TWH, van den Burg HA, Joosten MHAJ. Two for all: receptorassociated kinases SOBIR1 and BAK1. Trends Plant Sci. 2014;19:123-32.

74. Guzmán-Benito I, Donaire L, Amorim-Silva V, Vallarino JG, Esteban A, Wierzbicki AT, et al. The immune repressor BIR1 contributes to antiviral defense and undergoes transcriptional and post-transcriptional regulation during viral infections. New Phytol. 2019;224:421-38.

75. Leonetti P, Pantaleo V. Plant virus-derived siRNAs 'turn on' broad-spectrum plant immunity in wheat. Mol Plant. 2021;14:1038-40.

76. Murota K, Shimura H, Takeshita M, Masuta C. Interaction between Cucumber mosaic virus $2 \mathrm{~b}$ protein and plant catalase induces a specific necrosis in association with proteasome activity. Plant Cell Rep. 2017;36:37-47.

77. Hyodo K, Hashimoto K, Kuchitsu K, Suzuki N, Okuno T. Harnessing host ROS-generating machinery for the robust genome replication of a plant RNA virus. Proc Natl Acad Sci USA. 2017;114:E1282-90.

78. Wu J, Yang R, Yang Z, Yao S, Zhao S, Wang Y, et al. ROS accumulation and antiviral defence control by microRNA528 in rice. Nat Plants. 2017:3:16203

79. Liu P, Zhang X, Zhang F, Xu M, Ye Z, Wang K, et al. A virus-derived siRNA activates plant immunity by interfering with ROS scavenging. Mol Plant. 2021;14:1088-103.

80. Poirier EZ, Buck MD, Chakravarty P, Carvalho J, Frederico B, Cardoso A, et al. An isoform of Dicer protects mammalian stem cells against multiple RNA viruses. Science. 2021;373:231-6.

\section{Publisher's Note}

Springer Nature remains neutral with regard to jurisdictional claims in published maps and institutional affiliations. 